\title{
Frequency Dependence of HEMT Under Optical Illumination
}

\author{
Huang Yajian* \\ Arokiaswami Alphones`, Senior Member IEEE \\ *Department of Electrical Engineering 'School of Electrical and Electronic Engineering \\ National University of Singapore \\ Singapore, 119260 \\ Nanyang Technological University \\ Singapore, 639798
}

\begin{abstract}
An analysis of the AC characteristics of AlGaAs/GaAs HEMT under illumination with modulated light has been carried out for small signal condition. A new model for the photovoltage calculation is outlined. The effect of the signal frequency on the photoconductive current is evaluated, the results show that photoconductive current is very small and can be neglected in calculation. The frequency dependence of photovoltage along with 2-DEG charge density, drain-source current and transconductance of the device have been studied analytically for HEMT structure.
\end{abstract}

\section{INTRODUCTION}

The use of optical signals to control high speed microwave electronic circuits has been an area of growing interest because of their inherent isolation from RF signals. They can be used as an extra port to achieve various RF control funcitons such as gain control of amplifiers, oscillator tuning, locking and frequency modulation, as well as switching, mixing, limiting and phase shifting. Some authors have investigated the effect of optical illumination on the DC characteristics of MESFET's[1] and HEMT's[2], as well as the the AC characteristics of MESFET'S[3]. However, there is lack of study on the frequency dependent characteristics of HEMT under illumination with modulated light.

A detailed analysis of the AC characteristics of HEMT, considering all the physical mechanism due to the illumination, is a very complex task. However, by making some valid assumptions, a simple analysis considering the relevant photoeffects can be made. In AlGaAs/GaAs HEMT, photovoltaic effect and photoconducitve effect have been studied as the results of illumination[2]. In this paper, we use a different way from the traditional one [2] to calculate the photovoltage under illumination with modulated light. The contribution to the 2-DEG concentration increase due to photo-generated electron-whole pairs is also estimated, which explain the reason why the response due to photoconductive effect has not been observed clearly in AlGaAs/GaAs HEMT until now. Based on these, the frequency dependent I-V characteristics and transconductance characteristics are investigated, using a new method different from the work has been reported[4], in which only responsivity has been reported experimentally.

\section{THEORY}

Fig. 1 shows the band diagram of a typical depletion mode AlGaAs/GaAs HEMT under illumination with modulated light from top of the gate metal. The wavelenth of the signal carrier is shorter than the critical wavelength in AlGaAs $\left(\lambda_{\mathrm{C}}=h c / E g_{l}\right)$. The band-toband photo-absorption occurs both in the GaAs layer and the AlGaAs layer, generating hole-electron pairs in these regions. In the GaAs layer, the photo-generated electrons will be swept into the 2-DEG channel, which contributes to the increase of the electron concentration in the channel (photoconductive effect). In the AlGaAs layer, the photo-generated holes will be swept through the Schottky gate, which induces photovoltage(photovoltaic effect), and the photo-generated electrons will be swept into the 2-DEG channel, which increases the channel charge density (photoconductive effect). To calculate the AC performance of the AlGaAs/GaAs HEMT under illumination with the optical signal, considering the photovoltaic effect and photoconductive effect, the following assumption will be made: 1)the modulated light just covers the whole gatemetal surface; 2)the gate-metal is transparent to the incident light; 3)the photo-generated hole-electron pairs move at the saturation velocity when they are swept into 2-DEG channel or across the Schottky junction.

\section{A. Photovoltaic Effect}

The incident light flux density is assumed to be modulated by a signal of frequency w. Thus under small signal condition, the continuity equation for the number of photons incident onto the gate surface per second per unit area is $\phi(\omega)=\phi_{0}+\phi_{1} e^{j \omega t}$, where $\phi_{0}$ indicates the DC value and $\phi 1$ indicates the AC value. Assuming that the $\mathrm{DC}$ value is: $\operatorname{zero}\left(\phi_{0}=0\right)$, rms number of photons incident on the gate-metal surface per second per unit area is $\phi^{\prime}=\phi_{1} / \sqrt{2}$. In the AlGaAs layer, the photo-generated holes move along the reverse direction of z-axis, crossing the Schottky junction. The concentration of the holes, which crosses the metal-AlGaAs interface, at point $\mathrm{z}$ is given by[5]

$$
\Delta p(\omega, z)= \begin{cases}\eta \phi^{\prime}(1-R) \alpha_{1}\left(\frac{\tau_{\omega p 1}}{t_{r}(z)}\right) e^{-\alpha_{1} z} & z>d_{c 1} \\ \eta \phi^{\prime}(1-R) \alpha_{1} e^{-\alpha_{1} z} & z \leq d_{c 1}\end{cases}
$$

where $d_{c 1}=v_{s 1} \tau_{\omega p 1}$, is the critical length for AlGaAs, $R$ is the reflectivity efficiency of the metal surface, $\eta$ is the quantum efficiency, and $\alpha_{1}$ is the absorption efficiency in AlGaAs layer. The transit time $t_{r}(z)$ is defined as $z / v_{s}$, where $v_{s l}$ is the saturation hole velocity in AlGaAs layer. While $\tau_{\omega p 1}$ is the minory carrier hole 


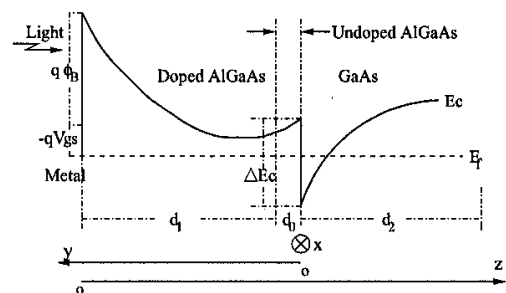

Fig. 1. Energy band diagram of AlGaAs/GaAs HEMT

lifetime at frequency $\omega$, which can be expressed as[5]

$$
\tau_{\omega p 1}=\frac{\tau_{p 1}}{\sqrt{1+\tau_{p 1}^{2} \omega^{2}}}
$$

where $\tau_{p l}$ is the minory carrier lifetime in DC condition in $\mathrm{AlGaAs}$ layer.

Integrating Eq.(1) from the metal-AlGaAs interface $(\mathrm{z}=$ $\mathrm{O})$ to the AlGaAs-GaAs interface $\left(z=d_{o}+d_{l}\right)$ and multiplying by $q$, the photocurrent density flowing across the Schottky junction is expressed as

$$
\begin{aligned}
J_{p h} & =q(1-R) \eta \phi^{\prime} \alpha_{1}\left(\int_{0}^{d_{c 1}} e^{-\alpha_{1} z} d z\right. \\
& \left.+\frac{v_{s 1} \tau_{p 1}}{\sqrt{1+\tau_{p 1}^{2} \omega^{2}}} \int_{d_{c 1}}^{d_{1}+d_{0}} \frac{e^{-\alpha_{1} z}}{z} d z\right)
\end{aligned}
$$

The photovoltage across the Schottky junction is obtained using the relation [3]

$$
V_{p h}(\omega)=\frac{K T}{q} \ln \left(\frac{J_{p h}}{J_{s}}\right)
$$

where $J_{s}$ is the reverse saturation current density across the Schottky junction, which can be expressed as[5]

$$
J_{s}=A^{*} T^{2} \exp \left(\frac{-q \phi_{B}}{K T}\right)
$$

where $\phi_{B}$ is the Schottky barrier height, and, $A^{*}=$ $(0.067+0.083 x) A$, is the effective Richardson constant for thermionic emission, neglecting the effects of optical phonon scattering and quantum mechanical reflection. For free electrons, the Richardson constant $A$ is $A=$ $120_{c m}^{-2} K^{-2}$. While $x$ is the Aluminium mole fraction of $A l_{x} G a_{l-x} A s$, which is 0.3 .

\section{B. Photoconductive Effect}

In the AlGaAs layer, the number of photo-generated electrons, being swept into the 2-DEG channel, equals to that of photo-generated holes moving across the Schottky junction. Therefore the charge flowing from the AlGaAs into the 2-DEG channel in unit time due to illumination is expressed as

$$
Q_{l}(w)=J_{p h} W L
$$

where $\mathrm{W}$ is the gate width of the HEMT, and L is the gate length of the HEMT.
Similarly, the charge flowing from the GaAs into the 2-DEG channel in unit time, due to illumination can be expressed as

$$
\begin{aligned}
& Q_{2}(\omega)=q W L(1-R) \eta \phi^{\prime} \alpha_{2} e^{-\alpha_{1}\left(d_{1}+d_{0}\right)} \\
& \quad\left(\int_{0}^{d_{c 2}} e^{-\alpha_{2} z} d z+\frac{v_{s 2} \tau_{p 2}}{\sqrt{1+\tau_{p 2}^{2} \omega^{2}}} \int_{d_{c 2}}^{d_{2}} \frac{e^{-\alpha_{2} z}}{z} d z\right)
\end{aligned}
$$

where $d_{c 2}=v_{s 2} \tau_{\omega p 2}, v_{s 2}$ is saturation electron velocity in the GaAs layer, and $\tau_{\omega p 2}$ is the minory carrier lifetime at frequency $\omega$ in the GaAs layer.

\section{I-V Characteristics Under Illumination}

To obtain the $I_{d s}-V g$ relation under illumination, the relation between 2-DEG electron concentration $n_{s}$ and the gate voltage $V_{g}$ must be determined. In this section, $n_{s}-V_{g}$ is numerically calculated from the solution of Poisson's equations. The AlGaAs layer is divided into two regions: the undoped region $0 \leq y<d_{0}$, and the doped region $d_{0} \leq y<d_{1}$ as shown in in Fig. 1 . In the doped AlGaAs layer, Poisson's equation under illumination is written as

$$
\frac{d^{2}\left(E_{c}(y)-E_{f}\right)}{d y^{2}}=\frac{q^{2}}{\epsilon}\left(N_{d}^{+}(y)-n(y)+p(\omega, y)\right)
$$

where $N_{d}^{+}$is the ionized donor concentration[6], n(y) is the free electron concentration[6], and $p(w, y)$ is the photo-generated hole concentration in this layer, which can be expressed as

$$
p(\omega, y)=\eta \phi^{\prime} \alpha_{1} e^{-\alpha_{1} y} \tau_{\omega p 1}(1-R)
$$

In the undoped region, the following boundary conditions are derived from the solution of Poisson's equation

$$
\begin{gathered}
E_{c f}\left(d_{0}\right)=\Delta E_{c}-E_{f}-\frac{q^{2} n_{s}}{\epsilon} d_{0} \\
\left.\frac{d E_{c f}(y)}{d y}\right|_{d_{0}}=-\frac{q^{2} n_{s}}{\epsilon}
\end{gathered}
$$

where $E_{c f}(y)=E_{c}(y)-E_{f}$ Using Taylor's series expansion, we can get

$$
\begin{array}{r}
E_{c f}(y+\Delta y)=E_{c f}(y)+\Delta y \frac{d E_{c f}(y)}{d y} \\
\frac{d E_{c f}(y+\Delta y)}{d y}=\frac{d E_{c f}(y)}{d y}+\Delta y \frac{d^{2} E_{c f}(y)}{d y^{2}}
\end{array}
$$

The above calculation is done iteratively from the doped and undoped region interface $(y=d o)$ to the gate metal $\left(y=d_{0}+d_{l}\right)$, where the gate voltage is dertmined by the final numerical solution

$$
V_{g}=-\frac{1}{q}\left(E_{c}\left(d_{0}+d_{1}\right)-E_{f}-\phi_{B}\right)
$$

There is a closed-form analytic expression[7], fitting the numerically calculated results, to determine the 2DEG electron density $n_{s}$ as a function of gate voltage $V_{g}$, which can be rewritten as

$$
n_{s}=n_{0}\left[\alpha+(1-\alpha) \tanh \left(\frac{V_{g}-V_{m}-V(x)}{V_{l}}\right)\right]
$$


where $V(x)$ is the channel potential ( $x$ is the coordinate parallel to the channel), $n_{0}, \alpha, V_{m}$ and $V_{l}$ are fitting parameters to be extracted from the numerically calculated data[7]. For a given gate and drain bias, the drain current is expressed as

$$
I_{d s}=q W n_{s}\left(V_{g}, V(x)\right) v(x)
$$

where $v(x)$ is the carrier velocity at point $x$. The velocity-field relation is obtained by[6],[7]

$$
v(x)= \begin{cases}\frac{\mu \frac{d V(x)}{d x}}{1+\frac{d}{E_{l}} \frac{d V(x)}{d x}} & V(x)<V_{s a t} \\ v_{s} & V(x) \geq V_{s a t}\end{cases}
$$

where $V_{\text {sat }}$ is the saturation potential corresponding to the saturation field $E$., and $E_{l}=E_{s} /\left(\eta E_{s} / v_{s}-1\right)$.

When the given drain voltage is in the linear region $\left(V_{d}<V_{\text {saa }}\right)$, substituting Eq.(17) into Eq. (16) and integrating from the source $(x=0)$ to the drain $(x=$ $L$ ), the drain current is found to be expressed as

$$
\begin{aligned}
I_{d s} & =\frac{q \mu Z n_{0}(1-\alpha) V_{l}}{L+V_{d} / E_{l}}\left[\frac{\alpha}{(1-\alpha) V_{l}} V_{d}\right. \\
& \left.-\ln \cosh \left(\frac{V_{g}-V_{m}-V_{d}}{V_{l}}\right)+\ln \cosh \left(\frac{V_{g}-V_{m}}{V_{l}}\right)\right]
\end{aligned}
$$

Similarly, for a given drain voltage is in saturation, the drain current can be expressed as

$$
\begin{aligned}
I_{d s}= & \frac{q \mu Z_{n_{0}}(1-\alpha) V_{l}}{L_{c}+V_{s a t}\left(E_{l}\right.}\left[\frac{\alpha}{(1-\alpha) V_{l}} V_{s a t}\right. \\
& \left.-\ln \cosh \left(\frac{V_{g}-V_{m}-V_{s a t}}{V_{l}}\right)+\ln \cosh \left(\frac{V_{g}-V_{m}-V_{d}}{V_{l}}\right)\right]
\end{aligned}
$$

where $x=L_{C}$ is the saturation point, and $L_{C}$ is the effective channel length in saturation[7]. The saturation voltage $V_{\text {sat }}$ in Eq. (19), can be obtained from the simultaneous solution of Eq. (16) and Eq. (18), assuming $x=L$ is the onset point of saturation $\left(V_{d}=V_{s a t}\right)$.

Now, to include the effect of illumination and the parasitic source $R_{s}$ and drain $R_{d}$ resistance, the gate and drain voltages in above equations are expressed in terms of terminal gate-source voltage $V_{g s}$, drain-source voltage $V_{d s}$ and photovoltage $V_{p h}$.

$$
\begin{aligned}
& V_{g}=V_{g s}+V_{p h}-I_{d s} R_{s} \\
& V_{d}=V_{d s}-I_{d s} R_{d}
\end{aligned}
$$

\section{Transconductance}

The transconductance of the device in the linear region $\left(V_{d}<V_{s a t}\right), g_{m L}$ is obtained by differentiating Eq.(18) with respect to $V_{g}$ at constant $V_{d}$

$$
g_{m L}=\frac{q \mu Z}{L+V_{d} / E_{l}}\left(n_{s 0}-n_{s L}\right)
$$

where $n_{s 0}$ is the 2-DEG electron density at $V(x)=O$, $n_{s L}$ is the 2-DEG electron density at $V(x)=V_{d}$.

While the device transconductance in the saturation region ( $\mathrm{vd} \geq \mathrm{V}_{\mathrm{sat}}$ ), $\mathrm{g}_{\mathrm{ns}}$ is is obtained by differentiating Eq.(19) with respect to $V_{g}$ at constant $V_{d}$

$$
\begin{aligned}
g_{m S}= & \frac{q \mu Z}{L_{c}+V_{s a t} / E_{l}}\left(n_{s 0}-\left(\frac{d V_{s a t}}{d V_{g}}\right) n_{s S}\right) \\
& -I_{d s S}\left(\frac{1}{E_{l}}+\frac{2 d_{s}}{E_{s} \cosh \pi\left(L-L_{c}\right)}\right) \frac{d V_{s a t}}{d V_{g}}
\end{aligned}
$$

where $\mathrm{n}_{8 \mathrm{~s}}$ is the 2-DEG electron density at $V(x)=V_{\text {sat }}$.

\section{RESULTS AND DISCUSSION}

Numerical calculations have been carried out for the photovolatge, the photo-generated charge into 2-DEG channel, 2-DEG charge density versus gate source voltage, the drian source current and the device transconductance under illumination with modulated li.qht. The device parameters and values of different constants used in the calculations are listed in Table III and its below

TABLE I

PARAMETERS USED IN THE SIMULATIONS

\begin{tabular}{c|c|c|c|c}
\hline \hline Region & thickness & $\begin{array}{c}\text { absorption } \\
\text { coefficency } \\
\left.\alpha(\mathrm{cm})^{-1}\right)\end{array}$ & $\begin{array}{c}\text { hole } \\
\text { lifetime } \\
\tau_{p}(\mathrm{~s})\end{array}$ & $\begin{array}{c}\text { saturatioy } \\
\text { velocity } \\
v_{s}(\mathrm{~cm} / \mathrm{s})\end{array}$ \\
\hline GaAs & 3000 & $1 \times 10^{4}$ & $10^{-8}$ & $8 \times 10^{6}$ \\
AlGaAs & 400 & $1.25 \times 10^{4}$ & $10^{-9}$ & $1 \times 10^{7}$ \\
$2 \mathrm{DEG}$ & 1400 & - & - & $1.85 \times 10^{7}$ \\
\hline \hline
\end{tabular}

gate width: $W_{G}=200 \mu m$, gate length: $L_{G}=0.5 \mu m$, the thickness of undoped AlGaAs: $d_{0}=25 \mathrm{~A}$, discontinuty in conduction band: $\Delta E_{c}=0.24 \mathrm{eV}$, Schottky barrier height: $\phi_{B}=0.95 \mathrm{~V}$.

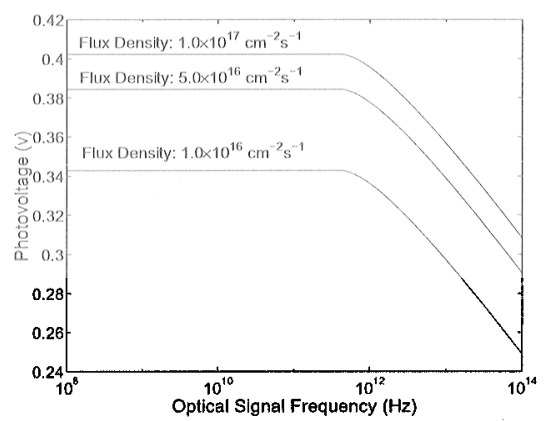

Fig. 2. photovoltage versus signal frequency for different flux densities

Fig. 2 shows the plots of photovoltages against the optical signal frequent incident flux densities. The photovoltage remains more or less constant and independent of signal frequency up to $500 \mathrm{GHz}$, which is much larger than that of MESFET'S (100 GHz) [3]. This is because the corner frequency (where the value begins to fall) of hole AC lifetime of HEMT's is about 10 times higher than that of MESFET'S. The reason for the photovoltage remains constant up to $500 \mathrm{GHz}$ is that the AC lifetime of holes is constant when frequency is much smaller than $1 / \tau_{p}$. The relative importance of the photoconductive effect can be deduced by Fig.3. The maximum value of the photo-generated charge swept into 2-DEG channel from AlGaAs and GaAs layers is less than $3 \times 10^{-9} \mathrm{C} / \mathrm{s}$. In other words, the maximum photoconductive current is less than $3 \times 10^{-6} \mathrm{rnA}$. Obviously, it is very difficult to observe the effect caused by the photoconductive current. Its importance can be neglected compared with the photovoltaic effect. The initial value for the AlGaAs is bigger than that for GaAs because it is much thicker than AlGaAs layer. While the corner frequency for ALGaAs is larger than that for GaAs because the hole lifetime in AlGaAs is shorter than that in GaAs. 


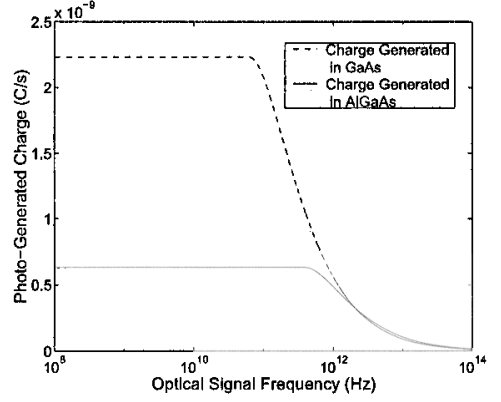

Fig. 3. Photo-generated charge against signal frequency

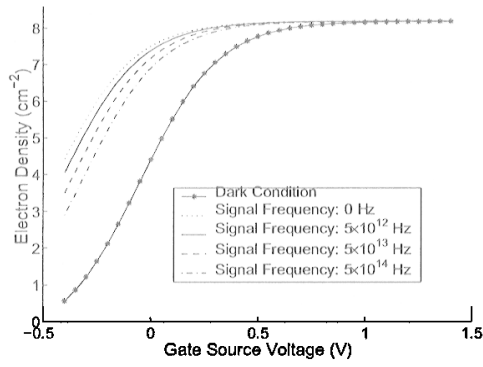

Fig. 4. 2-DEG electron density versus gate source voltage for different signal frequencies

Fig.4 represents the variation of 2-DEG charge density with terminal gate source voltage for different optical signal frequencies and dark condition. The results are calculated from the fitting Eq. (15). The following fitting parameters are extracted from the numerically calculated $n_{s}-V_{g s}$ curve, which is not presented in this paper: $\alpha=0.4643, n_{0}=8.2 \times 10^{11} \mathrm{~cm}^{-2}, V_{m}=$ $-0.0509 \mathrm{~V}, V_{i}=0.3686 \mathrm{~V}$. In dark condition, the results are similar to the observation already reported[7]. Under illumination, with the increase in signal frequency the channel charge density decreases, which corresponding to the AC $I-V$ characteristics of the device that is shown in Fig.5.

Fig.6 shows the transconductance of the device against the gate source voltage for different signal frequencies and dark situation. In dark situation, a optimum gate bias (about $0.1 \mathrm{~V}$ ) is observed which allowing the device operation at a maximum transconductance. At a particular value of $V_{g 8}$ the transconductance curve shifts to the left as the signal frequency decrease. This is clear from Fig.2 that the photovoltage decrease with the increase in signal frequency.

\section{CONCLUSION}

An analysis of the $\mathrm{AC}$ characteristics of $\mathrm{Al}$ GaAs/GaAs HEMT under illumination with modulated light has been carried out. The signal-modulated light is assumed to enter the device through the transparent gate metal only. Based on this, a new model for the photovoltage calculation is outlined. Results show that the corner frequency is much higher than that of MESFET's, which is due to the difference between the AC

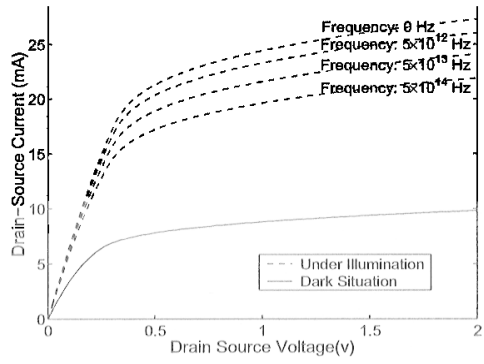

Fig. 5. I-V characteristics for different signal frequencies

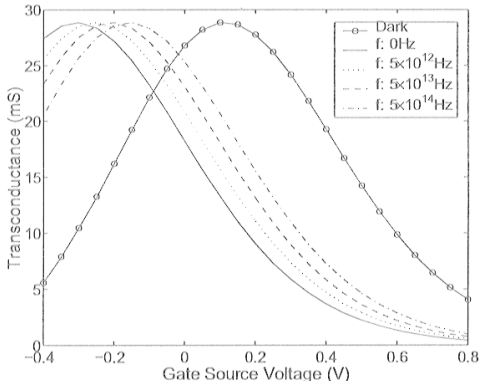

Fig. 6. Transconductance characteristics for different signal frequencies

carrier lifetime frequency characteristics in the two regions. The effect of the frequency on the photoconductive current is evaluated, considering the charge flowing from the $\mathrm{AlGaAs}$ and $\mathrm{GaAs}$ region into the 2-DEG channel. Although the signal frequency has significant effect on it, the results show that the relative value of the photoconductive current is very small comparing to drain to source current without illumination, which can be neglected in calculation. The $n_{s}-V_{p}, I-V$, and $g_{m}-V_{g s}$ curves show that the effects of signal frequency are all based on frequency dependent photovoltaic characteristics.

\section{REFERENCES}

[1] Alvaro Augusto A.de Salles, "Optical Control of GaAs MESFET's," IEEE Trans. Microwave Theory and Tech., vol. MTT31,no.10,pp.812-820, 0ctober 1983.

[2] Alvaro A,de Salles, Murilo A. Romero, "Alo,3Gao.7As/GaAs HEMT's Under Optical Illumination," IEEE Trans. Microwave Theory and Tech., voLMTT-39,no.12, pp.20102017, December 1991.

[3] Nandita Saha Roy, B. B.Pal, "Frequency-Dependent OPFET Characteristics with Improved Absorption under back Illumination", Journal of Lighttuave Technology, vol.18,no.4,pp.604613, April 2000.

[41 Yoshifumi Takanashi, Kiyoto Takahata and Yoshifumi Muramoto, "Characteristics 'of InAIAs/InGaAs High-ElectronMobilitv Transistors Under Illumination with modulated Light” ,“ IEEE Trans. Electron Device, voL46,no.12,pp.22712277,December 1999

[5] S. M.Sze "Physics of Semiconductor Devices", 1981.

[61 Abdollah Eskandarian, "Determination of the Small-Signal Parameters of an AlGaAs/GaAs MODFET", IEEE Trans Electron Device, vol.35,no.ll,pp.1793-1801,November 1988.

[7] M. Abdel Aziz, M. EI-Sayed and M.El-Banna, "An analytical model for small signal parameters in HEMTs including the effect of source/drain extrinsic resistances", Solid-State Electronics, 43,pp.891-900,January 1999. 